\title{
The yeast Wickerhamomyces anomalus (Pichia anomala) inhabits the midgut and reproductive system of the Asian malaria vector Anopheles stephensi
}

Irene Ricci, ${ }^{1}$ Claudia Damiani,, ${ }^{1}$ Patrizia Scuppa, ${ }^{1}$ Michela Mosca, ${ }^{1}$ Elena Crotti, ${ }^{2}$ Paolo Rossi, ${ }^{1}$ Aurora Rizzi, ${ }^{2}$ Aida Capone, ${ }^{1}$ Elena Gonella, ${ }^{3}$ Patrizia Ballarini, ${ }^{1}$ Bessem Chouaia, ${ }^{4}$ N' fale Sagnon, ${ }^{5}$ Fulvio Esposito, ${ }^{1}$ Alberto Alma, ${ }^{3}$ Mauro Mandrioli, ${ }^{6}$ Luciano Sacchi, ${ }^{7}$ Claudio Bandi, ${ }^{4}$ Daniele Daffonchio ${ }^{2}$ and Guido Favia ${ }^{1 *}$ ${ }^{1}$ Scuola di Bioscienze e Biotecnologie, Università degli Studi di Camerino, 62032 Camerino, Italy. ${ }^{2}$ Dipartimento di Scienze e Tecnologie Alimentari e Microbiologiche, Università degli Studi di Milano, 20133 Milan, Italy.

${ }^{3}$ Dipartimento di Valorizzazione e Protezione delle Risorse Agroforestali, Università degli Studi di Torino, 10095 Torino, Italy.

${ }^{4}$ Dipartimento di Patologia Animale, Igiene e Sanità Pubblica Veterinaria, Università degli Studi di Milano, 20133 Milan, Italy.

${ }^{5}$ Centre National de Recherche et de Formation sur le Paludisme (CNRFP), 01 B.P. 2208 Ouagadougou 01, Burkina Faso.

${ }^{6}$ Dipartimento di Biologia, Università degli Studi di Modena e Reggio Emilia, 41125 Modena, Italy. ${ }^{7}$ Dipartimento di Biologia Animale, Università degli Studi di Pavia, 27100 Pavia, Italy.

\section{Summary}

While symbiosis between bacteria and insects has been thoroughly investigated in the last two decades, investments on the study of yeasts associated with insects have been limited. Insect-associated yeasts are placed on different branches of the phylogenetic tree of fungi, indicating that these associations evolved independently on several occasions. Isolation of yeasts is frequently reported from insect habitats, and in some cases yeasts have been detected in the insect gut and in other organs/tissues. Here we show that the yeast Wickerhamomyces anomalus, previously known as Pichia anomala, is stably asso-

Received 24 November, 2009; accepted 7 November, 2010. *For correspondence. E-mail guido.favia@unicam.it; Tel. (+39) 0737 403230; Fax (+39) 0737403920. ciated with the mosquito Anopheles stephensi, a main vector of malaria in Asia. Wickerhamomyces anomalus colonized pre-adult stages (larvae $L_{1}-L_{4}$ and pupae) and adults of different sex and age and could be isolated in pure culture. By a combination of transmission electron microscopy and fluorescent in situ hybridization techniques, $W$. anomalus was shown to localize in the midgut and in both the male and female reproductive systems, suggesting multiple transmission patterns.

\section{Introduction}

Studies on the microbiome associated with haematophagus insects have mainly focused on the bacterial component rather than the eukaryotic one. In particular, only a few reports have been published on the yeast microbiota associated with mosquitoes. Ignatova and colleagues (1996) documented the isolation of Candida sp. from mosquitoes and more recently Pichia caribbica was isolated from the gut diverticulum of the dengue and yellow fever mosquito vector Aedes aegypti (Gusmão et al., 2007)

In recent years, the relationship between symbionts and mosquitoes has attracted a great deal of attention, for the perspective of exploiting the symbionts for blocking the transmission of parasites, for example, through the production of antagonistic factors (Favia et al., 2008; Moreira et al., 2009). 'Paratransgenesis' is defined as the genetic manipulation of midgut symbionts to express antiparasite effector molecules that could exert a direct action on the parasite (Riehle and Jacobs-Lorena, 2005). Yeast symbionts would offer an attractive alternative to bacteria for their potential to express antiparasite factors with folding properties suited to eukaryotic targets like the plasmodia.

We recently carried out research aimed at characterizing the microbiota in different mosquitoes involved in malaria transmission and identified bacteria of the genus Asaia as symbionts inhabiting the mosquito's midgut and gonads (Favia et al., 2007; Damiani et al., 2008; Crotti et al., 2009). At the microscope level, we observed that, beyond Asaia sp., yeast-like organisms were also present in the mosquito midgut and in reproductive systems. 


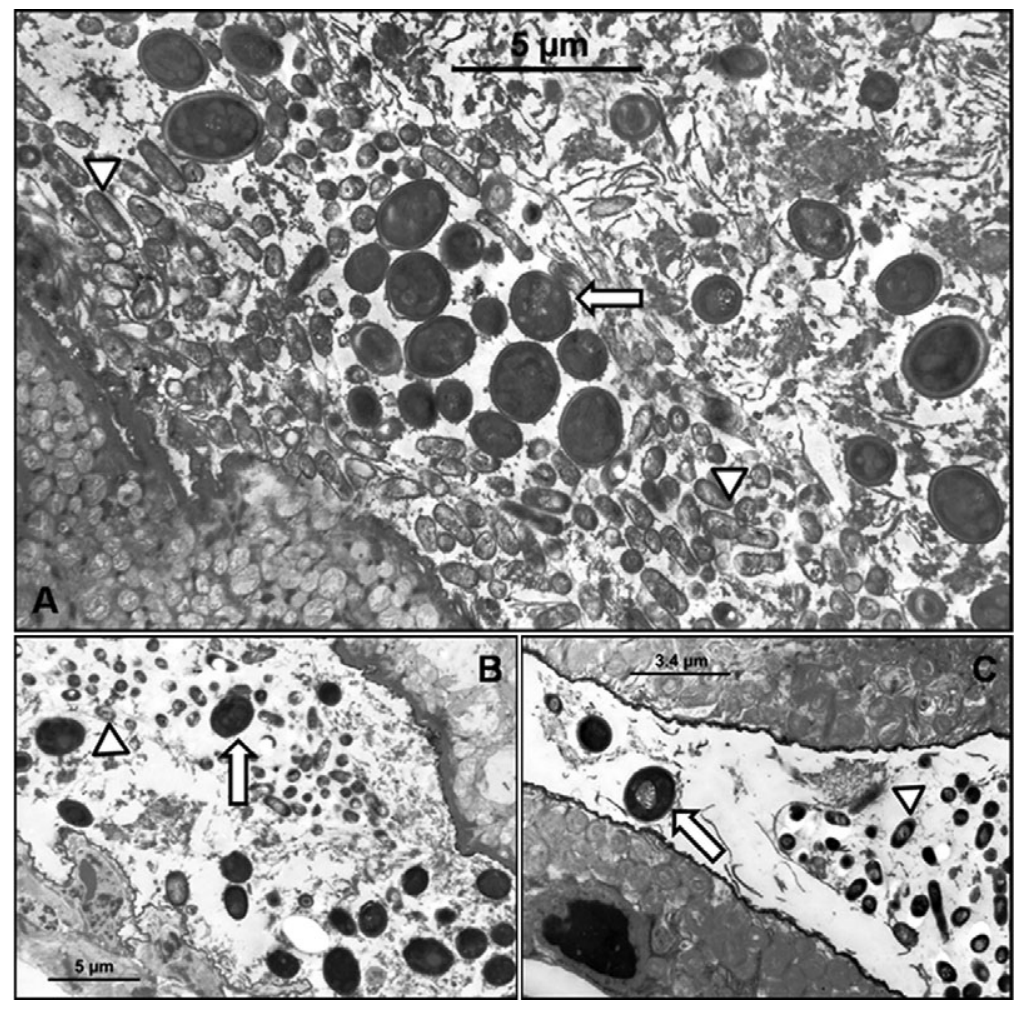

Fig. 1. Transmission electron microscopy micrographs of yeasts in Anopheles stephensi. Yeasts (arrows) and bacteria (triangles) are visible in the female mosquito midgut lumen $(A)$ and in the male gonoduct (B, C).

We report here the characterization of the yeasts associated with the malaria vector Anopheles stephensi (Diptera: Culicidae), identifying Wickerhamomyces anomalus (Saccharomycetales) as an important component of the yeast microbiome in this mosquito species.

\section{Results}

Analysis of yeasts in A. stephensi

Transmission electron microscopy analysis performed on mosquito specimens belonging to a laboratory-reared colony of $A$. stephensi, detected yeasts in the female midgut lumen and male gonoduct (Fig. 1). We frequently noticed yeasts in active division, especially at the level of the male reproductive system (Fig. 2), revealing that mosquito's tissues represent a favourable environment for these organisms. In order to identify these yeasts, we carried out an analysis of the mosquito-associated yeast community on both male and female individuals, using molecular and culture-dependent methods.

We identified six and three fungal clades/species, respectively, by culture-independent (Table 1) and plating analysis (Table 2). Twenty-two out of 34 sequences obtained by polymerase chain reaction (PCR)-based analysis from whole insects were phylogenetically related to Candida sp., Pichia sp. or W. anomalus according to 18S rRNA gene sequence analysis (Table 1). Twelve additional sequences matched with uncultured fungi from environmental samples and the fungal species Wallemia sebi, rarely associated with arthropods and never with insects. Wickerhamomyces anomalus-related sequences appeared to dominate in the gut followed by Pichia sp.

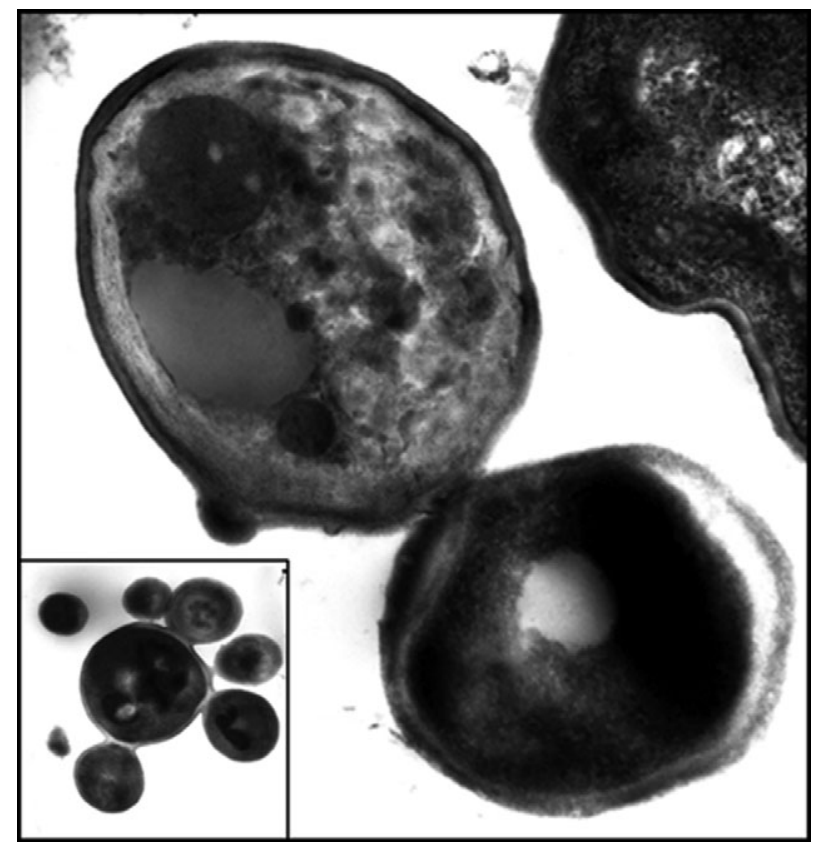

Fig. 2. Transmission electron microscopy micrographs revealing budding yeasts in active division in Anopheles stephensi male gonoducts. 
Table 1. Fungal sequences obtained from Anopheles stephensi by PCR-based screening with oligonucleotides designed on conserved sequences of $18 \mathrm{~S}$ rRNA.

\begin{tabular}{|c|c|c|c|c|c|c|c|c|c|c|}
\hline \multirow[b]{3}{*}{ Fungal identification (\% identity ${ }^{a}$ ) } & \multicolumn{9}{|c|}{ N. of clones/total clones for each DNA preparation } & \multirow{3}{*}{$\begin{array}{l}\text { N. of clones/ } \\
\text { total clones }\end{array}$} \\
\hline & \multicolumn{3}{|c|}{ Female individuals } & \multicolumn{3}{|c|}{ Male individuals } & \multicolumn{3}{|c|}{ Pools of female guts ${ }^{b}$} & \\
\hline & 1 & 2 & 3 & 1 & 2 & 3 & 1 & 2 & 3 & \\
\hline Candida sp. AY520257 (94-98\%) & $3 / 9$ & $1 / 6$ & $2 / 4$ & $1 / 7$ & $1 / 6$ & $2 / 2$ & $1 / 6$ & $1 / 4$ & $0 / 2$ & $12 / 46$ \\
\hline Pichia sp. AB022439 (95-98\%) & $2 / 9$ & $1 / 6$ & $1 / 4$ & $1 / 7$ & $3 / 6$ & $0 / 2$ & $2 / 6$ & $1 / 4$ & $0 / 2$ & $11 / 46$ \\
\hline Wickerhamomyces anomalus EF427893 (96-100\%) & $1 / 9$ & $1 / 6$ & $0 / 4$ & $1 / 7$ & $1 / 6$ & $0 / 2$ & $3 / 6$ & $2 / 4$ & $2 / 2$ & $11 / 46$ \\
\hline Wallemia sebi AF548107 (99-100\%) & $1 / 9$ & $1 / 6$ & $0 / 4$ & $1 / 7$ & $0 / 6$ & $0 / 2$ & $0 / 6$ & $0 / 4$ & $0 / 2$ & $3 / 46$ \\
\hline Uncultured fungus AM114819 (98-99\%) & $1 / 9$ & $1 / 6$ & $1 / 4$ & $2 / 7$ & $1 / 6$ & $0 / 2$ & $0 / 6$ & $0 / 4$ & $0 / 2$ & $6 / 46$ \\
\hline Uncultured fungus AB248107 (96-98\%) & $1 / 9$ & $1 / 6$ & $0 / 4$ & $1 / 7$ & $0 / 6$ & $0 / 2$ & $0 / 6$ & $0 / 4$ & $0 / 2$ & $3 / 46$ \\
\hline
\end{tabular}

a. \% identity range values of clones are reported per each fungal identification.

b. Each preparation consists of a pool of five guts.

Forty-six clones have been listed into six groups: Candida sp., Pichia sp., uncultured fungus (AM114819), uncultured fungus (AB248107), Wallemia sebi and Wickerhamomyces anomalus (total sequences and numbers of identified clones are reported per each group and sample).

and Candida sp. (Table 1). Analysis of Candida sp. and Pichia sp. sequences did not allow the identification at the species level.

Ten newly emerged lab-reared individuals (five males and five females) were examined for the diversity of the associated yeasts by cultivation. A total of 100 isolates have been collected and clustered according to the colony morphology and identified by sequencing the 18S-26S rRNA internal transcribed spacer (ITS) and the D1/D2 domain of the $26 \mathrm{~S}$ rRNA gene. Three yeast species have been identified: 77 isolates as Candida intermedia, 15 as Hanseniaspora uvarum and 8 as W. anomalus (Table 2).

The relatively small number of yeasts identified by both cultivation-dependent and independent approaches could reflect a limited diversity in the studied lab-reared colony (Rani et al., 2009) or could be due to the technical limitations of the approaches used, such as the choice of the cultivation media or the PCR primers (Zacchi and Vaughan-Martini, 2002; Hong et al., 2009).

The isolation of some Candida sp. from different mosquito species has been already documented (Ignatova et al., 1996). Candida intermedia, has been previously isolated from the gut of other insects (Nguyen et al., 2007). Yeast clades belonging to Pichia sp. have been previously associated to the intestinal tract of various insects (Nakase et al., 2007; Rao et al., 2007) including mosquitoes (Gusmão et al., 2007; 2010). However, Candida and Pichia appear to be ubiquitous in insects and were not associated with a specific host, which may suggest a non-exclusive relationship with mosquitoes. None of the sequences obtained by PCR-based analysis matched to the genus Hanseniaspora, even though we identified several isolates on plate as $H$. uvarum. Hanseniaspora strains have been isolated from insects belonging to different species (Pérez et al., 2003; Nguyen et al., 2007) supporting a wide distribution of these yeasts too.

Wickerhamomyces anomalus was identified both by molecular ecology analyses and by isolation. It was detected in both entire male and female mosquitoes, and in the female gut, where it has been revealed in all the samples. The observation that $W$. anomalus is not widely distributed among insects or insect habitats (Zacchi and Vaughan-Martini, 2002), suggested us that it may represent a symbiont specifically associated with $A$. stephensi and not just a transient commensal.

\section{Prevalence of $\mathrm{W}$. anomalus in A. stephensi and localization in mosquito organs}

Insects reared in near-axenic conditions have been analysed by both specific PCR tests and fluorescence in situ hybridization (FISH) for further investigating the presence of $W$. anomalus. A survey using a semi-nested PCR assay specific for $W$. anomalus was performed on $A$. stephensi individuals from the laboratory colony. Pre-adult stages and adults of different sex and age from three mosquito

Table 2. Yeast species associated to Anopheles stephensi identified following isolation in pure culture.

\begin{tabular}{llll}
\hline & & & GenBank Accession N. (\% identity) \\
\cline { 2 - 3 } N. isolates & Closest relative & ITS & D1/D2 \\
\hline 77 & Candida intermedia & FJ515166, (98-99\%) \\
15 & Hanseniaspora uvarum & FJ515178, (99\%) & FJ455102, (99-100\%) \\
8 & Wickerhamomyces anomalus & EU330185, (99-100\%) & FM \\
\hline
\end{tabular}

The percentages of identity of 18S-26S rRNA ITS and D1/D2 domain of the 26S rRNA gene are presented.

(C) 2011 Society for Applied Microbiology and Blackwell Publishing Ltd, Environmental Microbiology, 13, 911-921 
Table 3. Prevalence of Wickerhamomyces anomalus in different developmental stages and organs of Anopheles stephensi revealed by specific semi-nested PCR

\begin{tabular}{|c|c|c|c|c|}
\hline DNA source & $\begin{array}{l}\text { Developmental stages } \\
\text { (days post emergence) }\end{array}$ & $\begin{array}{l}\text { N. samples analysed per } \\
\text { mosquito generation }\end{array}$ & $\begin{array}{l}\text { N. of generations } \\
\text { examined }\end{array}$ & Positives/total \\
\hline \multirow[t]{6}{*}{ Whole mosquitoes } & Larvae- $L_{1}$ & 1 pool (15-20 individuals) & 3 & $3 / 3$ pools \\
\hline & Larvae- $\mathrm{L}_{2}$ & 1 pool (10 individuals) & 3 & $3 / 3$ pools \\
\hline & Larvae- $\mathrm{L}_{3}$ & 1 pool (5 individuals) & 3 & $3 / 3$ pools \\
\hline & Larvae- $\mathrm{L}_{4}$ & 15 individuals & 3 & 30/45 individuals \\
\hline & Pupae & 15 individuals & 3 & 29/45 individuals \\
\hline & Adults ( 0 day) & 50 individuals $\left(25 \%, 250^{7}\right)$ & 3 & $107 / 150$ individuals \\
\hline \multirow[t]{2}{*}{ o Guts } & Adults (2 days) & 1 pool (5 guts) & 3 & $3 / 3$ pools \\
\hline & Adults (10 days) & 1 pool (5 guts) & 3 & $3 / 3$ pools \\
\hline \multirow[t]{2}{*}{$O^{\prime \prime}$ Guts } & Adults (2 days) & 1 pool (5 guts) & 3 & $3 / 3$ pools \\
\hline & Adults (10 days) & 1 pool (5 guts) & 3 & $3 / 3$ pools \\
\hline \multirow[t]{2}{*}{ q Gonads } & Adults (2 days) & 1 pool (7 gonads) & 3 & $3 / 3$ pools \\
\hline & Adults (10 days) & 1 pool (7 gonads) & 3 & $3 / 3$ pools \\
\hline \multirow[t]{2}{*}{$\mathrm{O}^{x}$ Gonads } & Adults (2 days) & 1 pool (7 gonads) & 3 & $3 / 3$ pools \\
\hline & Adults (10 days) & 1 pool (7 gonads) & 3 & $3 / 3$ pools \\
\hline
\end{tabular}

Whole adult mosquitoes, larvae- $L_{4}$ and pupae have been tested individually, the earlier larval stages $\left(L_{1}, L_{2}, L_{3}\right)$ and dissected organs have been analysed as pools. Larval breeding water and adult sucrose solution have been used as negative controls for each PCR run. Number of dissected organs corresponded to 48 per generation and total of 144 mosquitoes.

generations were tested (Table 3). We examined larval stages $L_{1}-L_{3}$ and pupae as pools, and $L_{4}$ larvae and male and female adults as single individuals. Adults of different ages ( 0,2 and 10 days post emergence) were examined. The presence of $W$. anomalus was confirmed throughout the mosquito development and in the adult insect up to at least 10 days post emergence. To establish the prevalence of $W$. anomalus in adults, both female and male mosquitoes were sampled almost immediately after emergence and before sucrose solution was added to the cage. PCR experiments tested positive on all larvae and pupae pools (Table 3). The presence of $W$. anomalus was confirmed by PCR in $69 \%$ of the specimens tested individually, i.e. 30 positive $L_{4}$ larvae out of 45,29 out of 45 pupae, 107 out of 150 adults (Table 3).

Finding $W$. anomalus in newly emerged non-fed adults excluded the possibility that the yeast symbiont was acquired through food consumption or following a protracted permanence in the insectary. To clarify if the yeast can enter the environment and be ingested by the larvae, the breeding water was screened by the $W$. anomalusspecific PCR and tested negative.

Polymerase chain reaction analysis to identify yeast localization in specific tissues was performed on dissected organs from young (2 days post emergence) and old (10 days post emergence) adult mosquitoes in order to check for the stability of the association (Table 3). Newly emerged mosquitoes were allowed to feed on sterile sucrose solution for 2 days to eliminate meconium, thus preventing tissue contamination from possible leaking of the intestinal content during the organ dissection. A total of 24 organ preparations (eight per generation) were analysed consisting in guts and gonads from both males and females. Wickerhamomyces anomalus was detected in all the organ preparations analysed (Table 3). Particularly, the presence of the yeast in the gonads reinforced other evidences, i.e. detection in pre-adults and in newly emerged mosquitoes, that $W$. anomalus is not an occasional environmental contaminant and suggest that it could possibly undergo a vertical transmission route.

Wickerhamomyces anomalus localization in tissues found by semi-nested PCR analysis was confirmed by FISH experiments. FISH analysis indicated the localization of the yeast in the midgut of both female and male mosquitoes (Fig. 3) and in their gonads (Figs 4 and 5). Wickerhamomyces anomalus was particularly abundant in both the ovaries (Fig. 4) and the male reproductive system, displaying a wide distribution throughout the male apparatus, including testes, gonoducts and accessory glands (Fig. 5). Analysis of non-fed newly emerged individuals showed a weak fluorescence signal in comparison with older individuals (10 days post emergence), supporting the hypothesis that the yeast benefits of nutrients from the mosquito diet.

\section{Discussion}

The present study was conceived after transmission electron microscopy observation of yeasts in the gut and the reproductive system of $A$. stephensi. It was indeed their presence in the mosquito reproductive apparatus that suggested a specific association with the insect host, rather than an occasional presence, related for example to acquisition with food, or to other forms of acquisition from the environment. The subsequent molecular, microbiological and microscopy work that we performed on a laboratory-reared colony of $A$. stephensi confirmed that at 

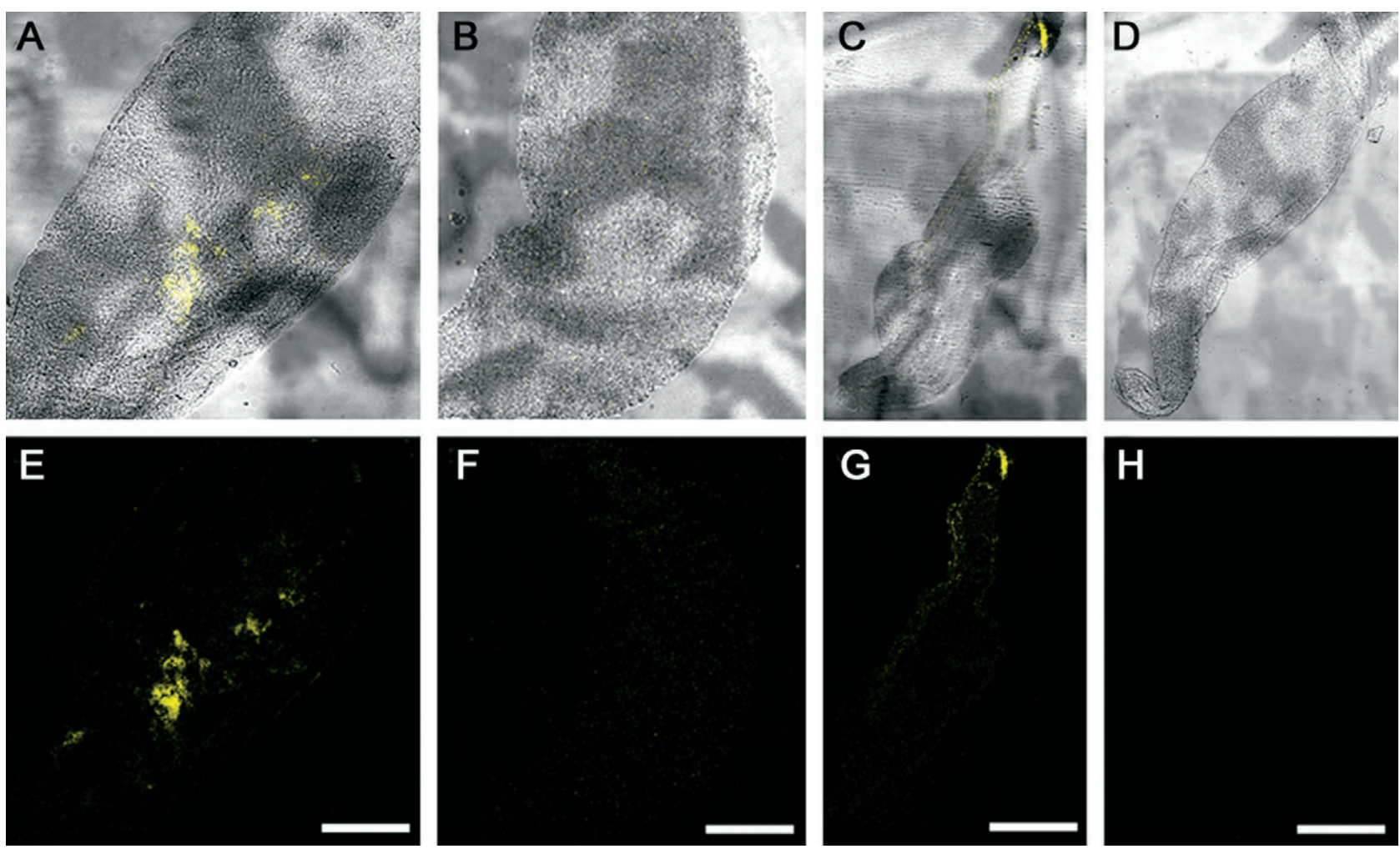

Fig. 3. FISH localization of Wickerhamomyces anomalus in Anopheles stephensi female (A, B) and male (C, D) midguts 10 days post emergence, observed by confocal laser scanning microscope analysis revealing bright signals (E, G). No fluorescence has been observed in the controls in the absence of the probes $(F, H)$. Bars correspond to $210 \mu \mathrm{m}$ in $\mathrm{A}, \mathrm{B}, \mathrm{E}, \mathrm{F}$ and to $140 \mu \mathrm{m}$ in $\mathrm{C}, \mathrm{D}, \mathrm{G}, \mathrm{H}$.

least one yeast species, W. anomalus, is specifically associated with these mosquitoes. The evidence for the association between $W$. anomalus and $A$. stephensi derives from the following results: (i) $W$. anomalus was identified by molecular and cultivation-based methods, (ii) W. anomalus was PCR-detected in mosquitoes from all development stages, with an average prevalence of about $70 \%$ in individuals tested at different ages and from different generations and (iii) $W$. anomalus was detected by using specific FISH probes in both male and female guts and reproductive systems.

The ascomycete $W$. anomalus, previously known as Pichia anomala (Kurtzman et al., 2008), is an heterothallic yeast, forming one to four shaped ascospores (Hansen, 1904), belonging to the so-called non-Saccharomyces wine yeasts (Rojas et al., 2003). Fredlund and colleagues (2002) reported that $W$. anomalus is physiologically versatile being capable of growing under a quite large $\mathrm{pH}$ range, under high osmotic pressure and anaerobic conditions, while it shows low tolerance to ethanol and acetate (Kalathenos et al., 1995).

The different portions of the gastrointestinal tract of adult mosquitoes have different $\mathrm{pH}$ according to the specific compartments. For example, $\mathrm{pH}$ is acidic in the lumen of the ventral diverticulum, named crop, that enlarges into the abdomen and which stores the sugar meal before slowly moving it to the midgut for the digestion step (Thompson, 1905). Moreover, Anopheles species are known to host in the gut acetic acid bacteria like Asaia (Favia et al., 2007; 2008; Crotti et al., 2009; Damiani et al., 2010) whose metabolism converts sugar to acids and that are capable to decrease the $\mathrm{pH}$ of the medium down to 3. Considering the quite wide variations of the environmental conditions in the gastrointestinal tract like $\mathrm{pH}$, redox potential, ionic strength and osmolarity, microbial symbionts living there should be capable to tolerate such environmental gradients. Yeasts like W. anomalus, that are capable of resisting different $\mathrm{pH}$, salinity and redox conditions, have high potential to be selected for living in the mosquito gut.

Wickerhamomyces anomalus has been isolated from a wide variety of habitats (Kurtzman and Dien 1998) and is considered a common spoilage organism in several products such as beer, silage, baking and dairy products (Kagiyama et al., 1988; Kitamoto et al., 1999; Dufour et al., 2003; Passoth et al., 2006). Although substantial work on the physiology and genetics of this yeast has been carried out (Passoth et al., 2006), there is a lack of information about its relationship with invertebrate organisms. In 2001, Kurtzman isolated this yeast from insect 

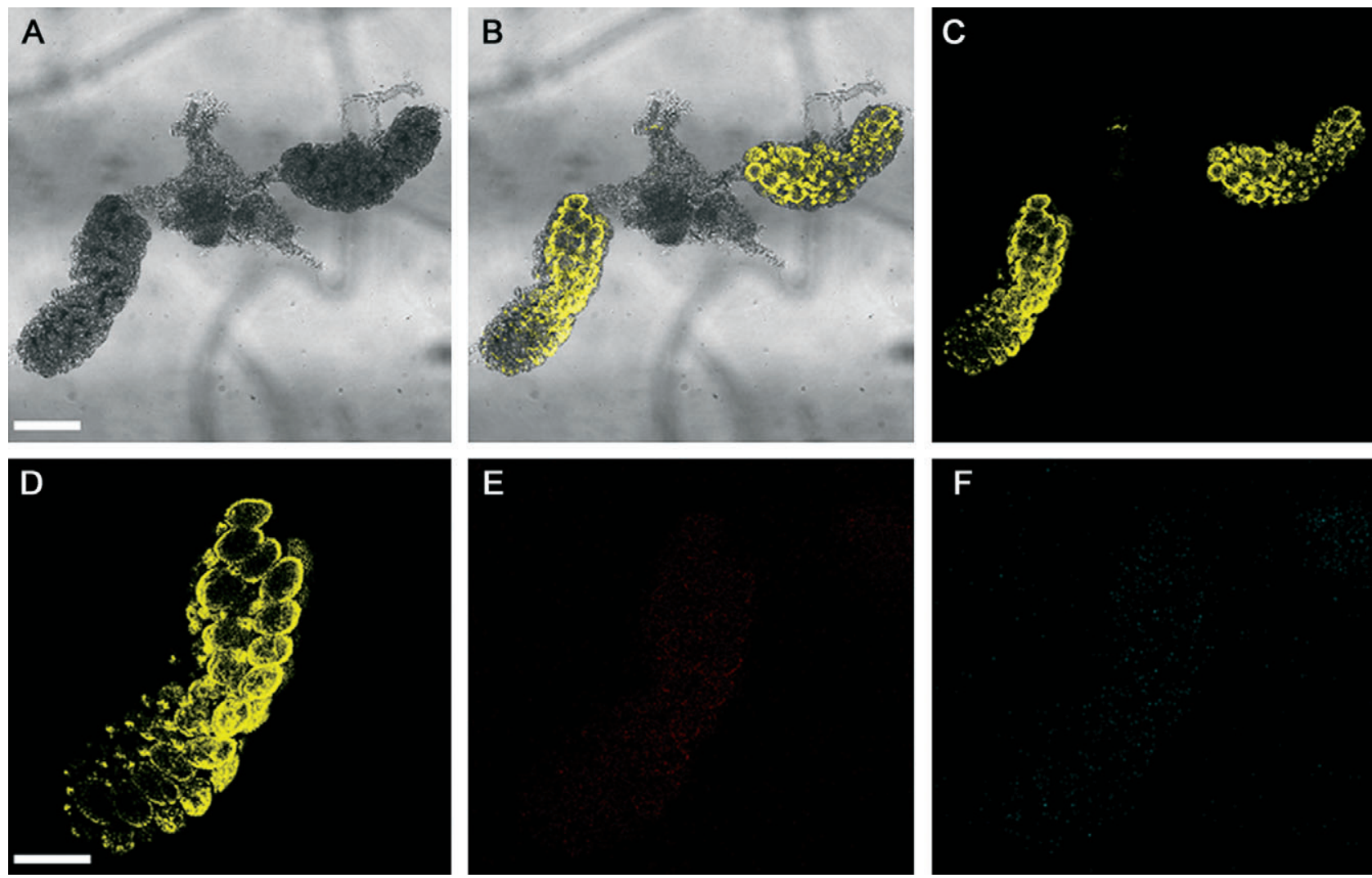

Fig. 4. FISH localization of Wickerhamomyces anomalus in Anopheles stephensi female gonad (A), observed by confocal laser scanning microscope analysis, revealed bright signals $(B, C)$. Magnification of an ovary portion (D-F) showed the presence of a strong fluorescence due to the $W$. anomalus-specific probes (D) and the absence of autofluorescence in the observed organ (E, F). Bars correspond to $280 \mu \mathrm{m}$ in $\mathrm{A}-\mathrm{C}$ and to $140 \mu \mathrm{m}$ in $\mathrm{D}-\mathrm{F}$.

frass from a Ponderosa pine (Kurtzman, 2001), and, more recently, a marine strain of $W$. anomalus has been identified as a killer yeast, acting against pathogenic fungi in the crab Portunus trituberculatus (Wang et al., 2007). Interestingly, a previous large screening for yeasts associated with different insects led to the detection of $W$. anomalus only in the inner body of Drosophila sp. (Zacchi and Vaughan-Martini, 2002). These authors suggested that while other Saccharomycetales (such as Pichia guilliermondii, $P$. membranifaciens, etc.) have an ubiquitous distribution among different insect species, not being associated with specific hosts, $W$. anomalus, having a more restricted distribution, could play a more specific biological role within the insect. Indeed, according to the wide environmental distribution of $W$. anomalus, that include plant material and natural sugar solutions, it might share environmental niches with larvae and adult mosquitoes, increasing the chance to be exposed to the insect body surface or to be ingested with the sugar-based meals.

The combination of the niche preference by $W$. anomaIus, together with its capacity to retain growth capability under the variable environmental conditions encountered in the mosquito gastrointestinal tract, makes this yeast pre-adapted to colonize the mosquito host body. The finding of $W$. anomalus not only in the gastrointestinal tract, but intimately associated to the mosquito reproductive systems, suggests that this yeast is not only ecologically pre-adapted, but can use some of its biological properties for moving among the different body compartments of the host, and has the capacity to escape the host immune barriers.

The presence of this yeast in mosquito and its location in specific organs raise questions on its functional role in the insect. Gut and gonads are characterized by the presence of nutrients available for yeasts and they represent excellent niches for nutritional mutualism, with supplementation of substances missing in carbohydrate diet of adult mosquitoes, as already described for other insects (Gibson and Hunter, 2009). Among the possible roles played by a symbiont like $W$. anomalus that should be explored in the future are the provision of nutrients to the host and its protection against pathogens (Dillon and Dillon, 2004; Moran et al., 2008). Considering that marine strains of $W$. anomalus have already been proposed to protect the host crab against pathogens (Wang et al., 

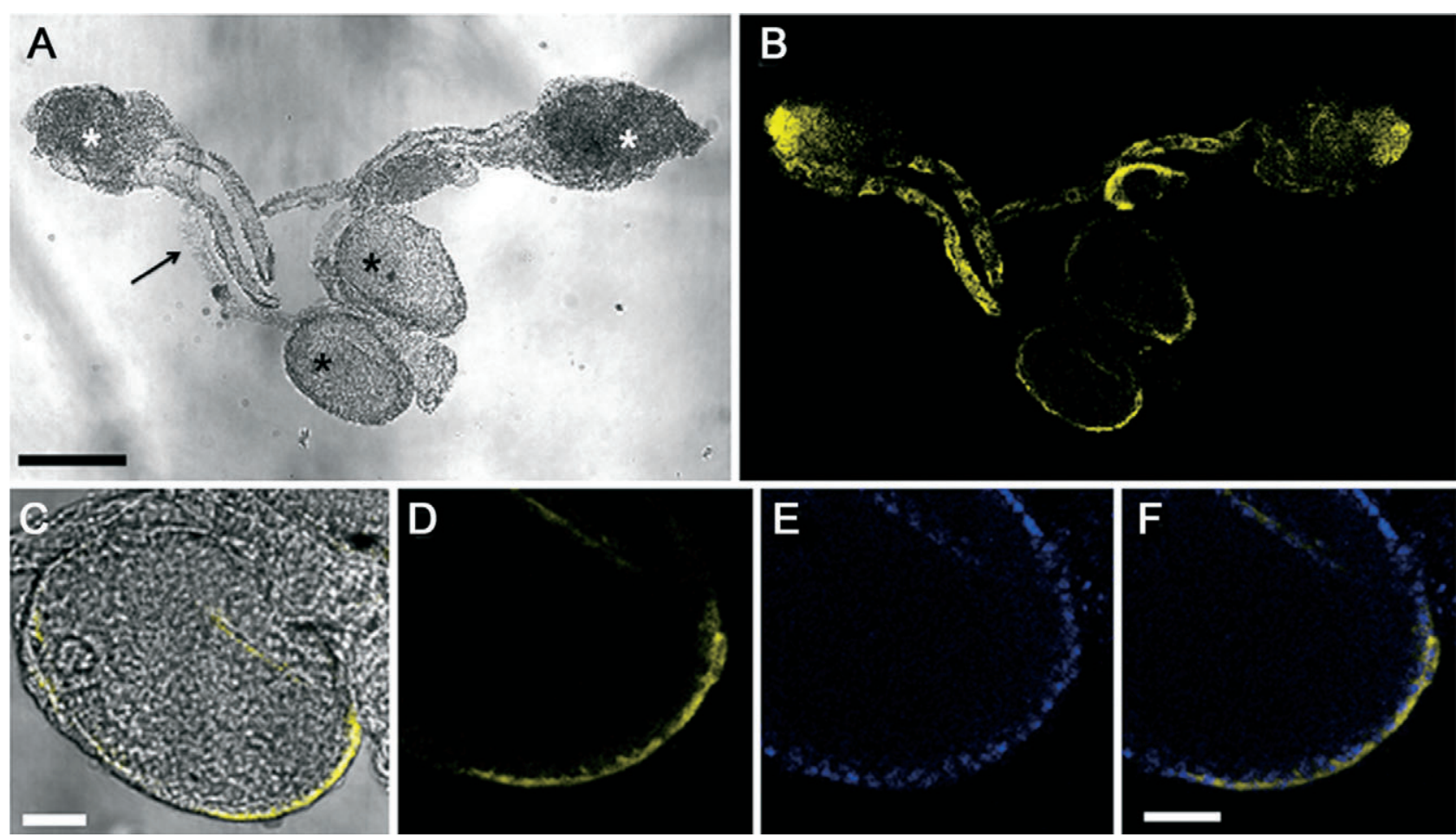

Fig. 5. FISH localization of Wickerhamomyces anomalus in Anopheles stephensi male reproductive system (A), observed by confocal laser scanning microscope analysis, reveals bright signals (B). White and black asterisks indicate testes and accessory glands respectively; arrow indicates gonoducts. Magnification of an accessory gland (C, D, F) showed a precise localization of the yeast (in yellow) at the periphery of the organ, as deduced also by DAPI staining (blue) of the nuclei (E, F). Bars correspond to $230 \mu \mathrm{m}$ in A, B and to $30 \mu \mathrm{m}$ in C-F.

2007; 2008), such a possibility should be also considered for $A$. stephensi.

Several reports indicate that bacteria and yeasts can localize both in the gut and in the reproductive apparatus of insect hosts (Noel and Atibalentja, 2006; Favia et al., 2007; Bressan et al., 2009). The localization of $W$. anomalus in the reproductive organs suggests a vertical transmission route through generations. Such a possibility is supported by the occurrence of $W$. anomalus in specimens from all life stages. Other transmission routes among adults could obviously be envisioned, including venereal transmission, as already reported for bacterial symbionts in A. stephensi (Favia et al., 2007; Damiani et al., 2008). Until a few decades ago, horizontal transmission was regarded as the most common route of transmission for yeasts associated with insects (Hagen et al., 1970). Several cases of yeast-insect associations have now been described, and maternal transmission of these symbionts is now frequently reported (Gibson and Hunter, 2005; 2009; Sacchi et al., 2008).

\section{Experimental procedures}

\section{Laboratory-reared mosquitoes}

Analysed mosquitoes were obtained from a colony of $A$. stephensi (Liston) reared for tens of years in the insectary of the Laboratory of Parasitology of the University of Camerino (Italy). They were maintained at standard setting of $30^{\circ} \mathrm{C}$ and $80 \pm 5 \%$ humidity, in near-axenic conditions during both the developmental and adult stages. After eggs eclosion, larvae were grown in clean tanks filled with deionized water containing sterile minced commercial mouse food. After rinsing, the pupae were transferred to clean dishes filled with sterile water and maintained in separate cages until adult emergence. Newly emerged mosquitoes not immediately analysed fed on sterilized $5 \%$ sucrose solution supplied on a hygienic cotton pad (renewed every $8 \mathrm{~h}$ ) for 2 days, thus allowing complete meconium evacuation before tissue dissection. Tissues from older mosquito (10 days post emergence) were analysed as well. Dissected organs were utilized for both DNA extraction and FISH analysis.

\section{Transmission electron microscopy}

Adults of $A$. stephensi were dissected in saline. Organs were harvested and prefixed in Karnovsky in cacodylate buffer (pH 7.2) (dos Santos and Gregório, 2002). After post-fixation in $2 \% \mathrm{OsO}_{4}$ for $1.5 \mathrm{~h}$, samples were washed in cacodylate buffer, dehydrated through an ethanol series, transferred in propylene oxide and embedded in Epon 812. Semi-thin sections were stained with $1 \%$ borate methylene blue and examined by light microscope. Thin sections were stained with saturated uranyl acetate, followed by Reynolds lead citrate and examined with a Zeiss EM 900 transmission electron microscope at $80 \mathrm{kV}$. 


\section{DNA extraction}

Before DNA extraction, adults mosquitoes were carefully washed as follows in order to sterilize the insect surface: samples were rinsed sequentially in sodium hypochlorite $(1 \%)$, chloridric acid $(0.5 \%)$, washed in $1 \times$ PBS between treatments and finally rinsed with $70 \%$ ethanol. Alcohol evaporation was allowed before further processing of the samples. In case of pre-adult stages, the specimens have been washed several times using $1 \times$ PBS and finally distilled water. DNA extraction was performed both from whole insects (all developmental stages) and adult specific organs. Dissections were carried out using sterile needles under a stereomicroscope, on a slide rinsed with a drop of sterile $1 \times$ PBS. To avoid cross-contamination among tissues, one anatomic portion from a single mosquito (gut or gonads) was recovered. DNA extraction was conducted under sterile conditions in a sterile cabinet (Bioair, Euroclone, Italy). Samples were homogenized in $50 \mu \mathrm{l}$ of digestion solution ( $50 \mathrm{mM}$ EDTA and $1.25 \mu \mathrm{l}$ of lyticase $0.5 \mathrm{mg} \mathrm{ml}^{-1}$ ) using sterile polypropylene pestles and incubated over night at $37^{\circ} \mathrm{C}$. After incubation, $100 \mu \mathrm{l}$ of extraction buffer (1\% SDS, $50 \mathrm{mM}$ Tris- $\mathrm{HCl} \mathrm{pH} \mathrm{8,} 25 \mathrm{mM} \mathrm{NaCl}, 25 \mathrm{mM}$ EDTA pH 8) was added to the samples and placed $30 \mathrm{~min}$ at $65^{\circ} \mathrm{C}$. Then $14 \mu \mathrm{l}$ of $8 \mathrm{M}$ potassium acetate $\mathrm{pH} 7.2$ was added; the homogenates were placed on ice for $30 \mathrm{~min}$ and then centrifuged for $10 \mathrm{~min}$ at 13000 r.p.m. DNA was precipitated in $100 \%$ ethanol. DNA pellets were dried and then rehydrated in $50 \mu \mathrm{l}$ of ultra-pure water for PCR applications and the nucleic acid concentration has been estimated by spectrophotometer NanoDrop 1000 .

\section{Yeast identification by PCR-based analysis}

Polymerase chain reaction screening for mosquitoassociated yeasts was performed using the primers yeastF1 (5'-TAATTCTAGAGCTAATACATG- $\left.3^{\prime}\right)$ and yeast-R1 (5'-GGAAACGTCCTTGGCAAA-3') designed on conserved sequences of the locus $18 \mathrm{~S}$ rRNA resulting from the alignment of yeasts from different orders. The genomic DNA from six whole mosquitoes (three males and three females) and three female gut preparations (five guts each) was used as template for the PCR amplifications; to avoid meconium leak, guts were dissected 2 days post emergence, whereas whole mosquitoes were analysed at the emergence and before the meal. Each PCR reaction was carried out, under sterile conditions, in a total volume of $25 \mu$ l containing: $1 \times$ PCR Gold Buffer, $2.5 \mathrm{mM} \mathrm{MgCl}, 0.25 \mathrm{mM}$ dNTPs, $0.2 \mu \mathrm{M}$ of each primer, $1 \cup$ Ampli Taq Gold (Applied Biosystems, USA) and $10 \mathrm{ng}$ template DNA. PCR was carried on a Veriti Thermal Cycler (Applied Biosystems, USA) under these conditions: $94^{\circ} \mathrm{C} / 10 \mathrm{~min} ; 30 \times\left(94^{\circ} \mathrm{C} / 30 \mathrm{~s}, 50^{\circ} \mathrm{C} / 45 \mathrm{~s} \Delta t+0.2^{\circ} \mathrm{C}, 72^{\circ} \mathrm{C} /\right.$ $45 \mathrm{~s}) ; 72^{\circ} \mathrm{C} / 10 \mathrm{~min}$. After amplification, $5 \mu \mathrm{l}$ of PCR products were visualized by electrophoresis on ethidium bromide agarose gel (1.5\%) under UV light. Remaining volumes were purified by centrifugation in $1 \mathrm{~V}$ of isopropanol, rehydrated in $10 \mu \mathrm{l}$ of water and cloned using the pGem-T Easy Vector System kit (Promega, USA) in Escherichia coli JM109 competent cells. Purified recombinant plasmids were sequenced at the MWG-Genomic Company (Ebersberg, Germany). Sequences were compared with the database at the National Centre for Biotechnology Information by using BLAST (http:// blast.ncbi.nlm.nih.gov/Blast.cgi).

\section{Yeast isolation and characterization}

To isolate yeasts associated with $A$. stephensi, two different media, universally used for the isolation and cultivation of yeasts, were used: YM agar medium (Difco, Milano, Italy) and modified PDB liquid medium (glucose $20 \mathrm{~g} \mathrm{l}^{-1}$, yeast extract $10 \mathrm{~g} \mathrm{l}^{-1}$, peptone $10 \mathrm{~g} \mathrm{l}^{-1}$, potato extract $8 \mathrm{~g} \mathrm{l}^{-1}$, $\mathrm{pH}$ 5.7). Ten newly emerged non-fed mosquitoes (five females and five males) were surface sterilized in $100 \%$ ethanol and washed twice in $0.9 \% \mathrm{NaCl}$. The insects were individually homogenized, with a sterile pestle, by grinding in $200 \mu \mathrm{l}$ of $0.9 \%$ $\mathrm{NaCl}$. Approximately $100 \mu \mathrm{l}$ of each homogenate was spread directly on YM agar plates supplemented with rifampicin $\left(40 \mu \mathrm{g} \mathrm{ml}^{-1}\right)$ to avoid bacterial growth. The plates were incubated overnight at $30^{\circ} \mathrm{C}$. The remaining half homogenate was inoculated in modified PDB medium and grown overnight at $30^{\circ} \mathrm{C}$. After growth, the microorganisms were spread on YM agar and incubated overnight at $30^{\circ} \mathrm{C}$. Yeast colonies were categorized and selected based on colony morphology and 100 pure cultures of yeast strains were stored in $15 \%$ glycerol at $-80^{\circ} \mathrm{C}$.

18S-26S rRNA ITS and 26S rRNA gene analyses were carried out for all the isolated yeasts. ITS fragments covering ITS1, 5.8S rRNA gene and ITS2; together with the D1/D2 region of 26S rRNA gene are usually used for yeast identification (Manter and Vivanco, 2007; Ferreira et al., 2010). For the amplification of ITS fragment, the primers used were ITS1F (5'-CTTGGTCATTTAGAGGAAGTAA- $\left.3^{\prime}\right)$ and ITS4 (5'-TCCTCCGCTTATTGATATGC-3') under the following amplification conditions in a final volume of $50 \mu \mathrm{l}$ : $1 \times$ Taq polymerase buffer, $1.8 \mathrm{mM} \mathrm{MgCl}_{2}, 0.2 \mathrm{mM}$ dNTPs, $0.5 \mu \mathrm{M}$ of each primer and $2 \mathrm{U}$ of Taq DNA polymerase (Invitrogen, Milan, Italy). Thermal conditions of PCR reactions were as follows: a denaturation step of $7 \mathrm{~min}$, followed by 35 cycles of $45 \mathrm{~s}$ at $94^{\circ} \mathrm{C}, 45 \mathrm{~s}$ at $55^{\circ} \mathrm{C}$ and $1 \mathrm{~min}$ at $72^{\circ} \mathrm{C}$, with a final extension step of $10 \mathrm{~min}$ at $72^{\circ} \mathrm{C}$ (Sun et al., 2009). PCR amplification of the D1/D2 domain of 26S rRNA gene was carried out using primers NL1 (5'-GCATATCAATAAGCG GAGGAAAAG-3') and NL4 (5'-GGTCCGTGTTTCAAGAC GG-3'). PCR was performed using $1 \times$ Taq polymerase buffer, $1.8 \mathrm{mM} \mathrm{MgCl}, 0.2 \mathrm{mM}$ dNTPs, $0.5 \mu \mathrm{M}$ of each primer and $2 \mathrm{U}$ of Taq DNA polymerase (Invitrogen) in a final volume of $50 \mu$ l. The reaction conditions were: $7 \mathrm{~min}$ at $94^{\circ} \mathrm{C}, 35$ cycles of $45 \mathrm{~s}$ at $94^{\circ} \mathrm{C}, 1 \mathrm{~min}$ at $59^{\circ} \mathrm{C}, 1 \mathrm{~min}$ at $72^{\circ} \mathrm{C}$ and a final extension step of $10 \mathrm{~min}$ at $72^{\circ} \mathrm{C}$ (Sun et al., 2009). Amplicons obtained from ITS and 26S rRNA gene PCRs were clustered according to the fingerprinting profile. Representative amplicons from each group were sequenced, analysed and aligned using the BLAST program in the National Centre for Biotechnology Information database.

\section{W. anomalus-specific semi-nested PCR assay}

Semi-nested PCR amplifications, specific for $W$. anomalus, were carried out on three generations (every second generation) of our laboratory-reared colony of $A$. stephensi, using three oligonucleotides designed on the yeast sequence isolated from mosquitoes, which shared $100 \%$ homology with the locus $18 \mathrm{~S}$ rRNA of $W$. anomalus present in the database with Accession Number EF427893. The oligonucleotides employed were Wa-for (5'-GTGAAACTGCGAATGGCTCAT 
TAAATC-3'), Wa-rev (5'-GCCTTCCTTGGATGTGGTAGC-3') and Wa-in (5'-TTTTCGAATCGCATGACTTCGTGTC-3'). The outer oligos (Wa-for and Wa-rev) were designed on conserved sequences of the gene 18S rRNA, whereas the inner oligo (Wa-in) is $W$. anomalus-specific; primers have been chosen after the sequences alignment analysis of different Saccharomycetales by the ClustalW program (http://www. ebi.ac.uk/Tools). Amplification pattern included two PCR products, of, respectively, $356 \mathrm{bp}$ and $167 \mathrm{bp}$, obtained from both isolate pure cultures (used as positive controls) and mosquito metagenomic DNA; randomly chosen 167 bp products have been sequenced and confirmed to be $W$. anomalus-specific. PCR reactions were carried out as previously described (see PCR-based method), except for the oligos concentration that was $0.2 \mu \mathrm{M}$ Wa-for, $0.5 \mu \mathrm{M}$ Wa-in and $0.6 \mu \mathrm{M}$ Wa-rev. PCR amplification was performed in a Veriti Thermal Cycler (Applied Biosystems, USA) under these conditions: $94^{\circ} \mathrm{C} / 10 \mathrm{~min}$; $35 \times\left(94^{\circ} \mathrm{C} / 30 \mathrm{~s} ; 60^{\circ} \mathrm{C} / 30 \mathrm{~s} ; 72^{\circ} \mathrm{C} /\right.$ $30 \mathrm{~s}) ; 72^{\circ} \mathrm{C} / 7 \mathrm{~min}$. In each amplification larval breeding water and sucrose solution were used as negative controls. Approximately $150 \mathrm{ml}$ of larval breeding water at $\mathrm{L}_{4}$ stage (around 1 week after the eggs transfer into the tank) was filtered by a Whatman filter paper. Filters were then washed twice in sterile $1 \times$ PBS and the recovered PBS was centrifuged at maximum speed for $10 \mathrm{~min}$. DNA from the pellets was extracted using the protocol previously described.

\section{W. anomalus localization in A. stephensi by FISH}

For whole mount FISH, analysis on pure yeast cultures was performed to test the specificity level of the designed probes; $W$. anomalus and $C$. intermedia strains (the last being a negative control) isolated from $A$. stephensi were employed. Once the probes were confirmed to be target-specific and the experimental conditions were established, we carried out experiments on dissected organs from both male and female mosquitoes (newly emerged and 10 days post emergence). Hybridization was carried out on guts and gonads using two $W$. anomalus-specific fluorescent probes targeting the 18S rRNA gene: Wa1 (5'-TATGAATCATCAAAGAGCTCAGA AGA-3') and Wa2 (5'-GACACGAAGTCATGCGATTCGA $\left.A A A-3^{\prime}\right)$. Probes were labelled at the $5^{\prime}$ end with fluorochrome Cy5 (indodicarbocyanine, absorption/emission at $650 / 670 \mathrm{~nm}$ ). A FISH protocol was adapted from Xufre and colleagues (2006), with $2 \mathrm{~h}$ hybridization at $40^{\circ} \mathrm{C}$. Before hybridization, dissected tissues were fixed overnight at $4^{\circ} \mathrm{C}$ in ethanol/1× PBS $(1: 1)$, then $5 \mathrm{~min}$ at $4^{\circ} \mathrm{C}$ in $4 \%$ paraformaldehyde and washed in $1 \times$ PBS. Dissected organs were then incubated for $10 \mathrm{~min}$ at a temperature of $37^{\circ} \mathrm{C}$ with a $10 \mu \mathrm{g} \mathrm{ml}^{-1}$ pepsin solution and washed again in $1 \times$ PBS. Hybridization was conducted in dark conditions at $40^{\circ} \mathrm{C}$ for $2 \mathrm{~h}$, with $100 \mu \mathrm{l}$ of hybridization buffer (2x SSC, $50 \%$ formamide, $1 \%$ dextran sulfate, $0.1 \mu \mathrm{g} \mathrm{ml}^{-1}$ probes). After hybridization, dissections were washed in $100 \mu \mathrm{l}$ of washing buffer ( $2 \times \mathrm{SSC}, 50 \%$ formamide, $1 \%$ dextran sulfate) at $40^{\circ} \mathrm{C}$ for $15 \mathrm{~min}$. Subsequently two washes in $0.1 \times$ SSC (at room temperature for $10 \mathrm{~min}$ ) and then two washes in $1 \times$ SSC (at room temperature for $10 \mathrm{~min}$ ) were performed, followed by two washes in $1 \times \mathrm{PBS}$ at $37^{\circ} \mathrm{C}$. Then incubation with $50 \mathrm{ng}$ of DAPI (4', 6'-diamidino-2-phenylindole) for $5 \mathrm{~min}$ at room temperature was performed, followed by two washes in $1 \times$
PBS for $5 \mathrm{~min}$ at room temperature. Finally, samples were mounted in anti-fading medium then observed using laser scanning confocal microscope SP2-AOBS (Leica). Control experiments involved treatment of slides in absence of probes.

\section{Acknowledgements}

I.R. was funded by 'Compagnia di San Paolo' in the context of the Italian Malaria Network. E.C and P.R. received a travel grant from the COST Action FA0701. The project was supported by Firb-Ideas (Grant RBID082MLZ) and Prin 2007 (Grant 2007PK2HB7-002) both from the Italian Ministry of Education, University and Research (MIUR) to G.F. D.D. thank for financial support the European Union in the ambit of project BIODESERT (European Community's Seventh Framework Programme CSA-SA REGPOT-2008-2 under Grant Agreement No. 245746). C.B., E.C and D.D. benefited of travel grants from Cost Action FA0701: 'Arthropod Symbiosis: From Fundamental Studies to Pest and Disease Management'.

\section{References}

Bressan, A., Arneodo, J., Simonato, M., Haines, W.P., and Boudon-Padieu, E. (2009) Characterization and evolution of two bacteriome-inhabiting symbionts in cixiid planthoppers (Hemiptera: Fulgoromorpha: Pentastirini). Environ Microbiol 11: 3265-3279.

Crotti, E., Damiani, C., Pajoro, M., Gonella, E., Rizzi, A., Ricci, I., et al. (2009) Asaia, a versatile acetic acid bacterial symbiont, capable of cross-colonizing insects of phylogenetically distant genera and orders. Environ Microbiol 11: 3252-3264.

Damiani, C., Ricci, I., Crotti, E., Rossi, P., Rizzi, A., Scuppa, P., et al. (2008) Paternal transmission of symbiotic bacteria in malaria vectors. Curr Biol 18: R1087-R1088.

Damiani, C., Ricci, I., Crotti, E., Rossi, P., Rizzi, A., Scuppa, P., et al. (2010) Mosquito-bacteria symbiosis: the case of Anopheles gambiae and Asaia. Microb Ecol 60: 644654.

Dillon, R.J., and Dillon, V.M. (2004) The gut bacteria of insects: non-pathogenic interactions. Annu Rev Entomol 49: 71-92.

Dufour, J.P., Verstrepen, K., and Derdelinckx, G. (2003) Brewing yeasts. In Yeasts in Food - Beneficial and Detrimental Aspects. Boekhout, T., and Robert, V. (eds). Hamburg, Germany: Behr's Verlag, pp. 389-412.

Favia, G., Ricci, I., Damiani, C., Raddadi, N., Crotti, E., Marzorati, M., et al. (2007) Bacteria of the genus Asaia stably associate with Anopheles stephensi, an Asian malarial mosquito vector. Proc Natl Acad Sci USA 104: 9047-9051.

Favia, G., Ricci, I., Marzorati, M., Negri, I., Alma, A., Sacchi, L., et al. (2008) Bacteria of the genus Asaia: a potential paratransgenic weapon against malaria. Adv Exp Med Biol 627: 49-59.

Ferreira, N., Belloch, C., Querol, A., Manzanares, P., Vallez, S., and Santos, A. (2010) Yeast microflora isolated from brazilian cassava roots: taxonomical classification based on molecular identification. Curr Microbiol 60: 287-293. 
Fredlund, E., Druvefors, U., Boysen, M.E., Lingsten, K.J., and Schnürer, J. (2002) Physiological characteristics of the biocontrol yeast Pichia anomala J121. FEMS Yeast Res 2: 395-402.

Gibson, C.M., and Hunter, M.S. (2005) A reconsideration of the role of yeast associated with Chrysoperla lacewings. Biol Control 32: 57-64.

Gibson, C.M., and Hunter, M.S. (2009) Negative fitness consequences and transmission dynamics of a heritable fungal symbiont in a parasitic wasp. Appl Environ Microbiol 75: 3115-3119.

Gusmão, D.S., Santos, A.V., Marini, D.C., Russo Ede, S., Peixoto, A.M., Bacci Júnior, M., et al. (2007) First isolation of microorganisms from the gut diverticulum of Aedes aegypti (Diptera: Culicidae): new perspectives for an insect-bacteria association. Mem Inst Oswaldo Cruz 102: 919-924.

Gusmão, D.S., Santos, A.V., Marini, D.C., Bacci, M., Jr, Berbert-Molina, M.A., and Lemos, F.J. (2010) Culturedependent and culture-independent characterization of microorganisms associated with Aedes aegypti (Diptera: Culicidae) (L.) and dynamics of bacterial colonization in the midgut. Acta Trop 115: 275-281.

Hagen, K.S., Tassan, R.L., and Sawall, E.F. (1970) Some ecophysiological relationships between certain Chrysopa, honeydews and yeasts. Bull Lab Entomol Agric fil Silv 28: 113-134.

Hansen, E.C. (1904) Grundlinien zur Systematik der Saccharomyceten. Zentralbl Bakteriol Parasitenkd 12: 529-538.

Hong, S., Bunge, J., Leslin, C., Jeon, S., and Epstein, S.S. (2009) Polymerase chain reaction primers miss half of rRNA microbial diversity. ISME J 3: 1365-1373.

Ignatova, E.A., Nagornaia, S.S., Povazhnaia, T.N., and lanishevskaia, G.S. (1996) The yeast flora of blood-sucking mosquitoes. Mikrobiol Z 58: 12-15.

Kagiyama, S., Aiba, T., Kadowaki, K., and Mogi, K. (1988) New killer toxins of halophilic Hansenula anomala. Agric Biol Chem 52: 1-7.

Kalathenos, P., Baranyi, J., Sutherland, J.P., and Roberts, T.A. (1995) A response surface study on the role of some environmental factors affecting the growth of Saccharomyces cerevisiae. Int J Food Microbiol 25: 63-74.

Kitamoto, H.K., Hasebe, A., Ohmomo, S., Suto, E.G., Muraki, M., and IImura, Y. (1999) Prevention of aerobic spoilage of maize silageby a genetically modified killer yeast, Kluyveromyces lactis, defective in the ability to grow on lactic acid. Appl Environ Microbiol 65: 4697-4700.

Kurtzman, C.P. (2001) Four new Candida species from geographically diverse locations. Antonie Van Leeuwenhoek 79: 353-361.

Kurtzman, C.P., and Dien, B.S. (1998) Candida arabinofermentans, a new L-arabinose fermenting yeast. Antonie Van Leeuwenhoek 74: 237-243.

Kurtzman, C.P., Robnett, C.J., and Basehoar-Powers, E. (2008) Phylogenetic relationships among species of Pichia, Issatchenkia and Williopsis determined from multigene sequence analysis, and the proposal of Barnettozyma gen. nov., Lindnera gen. nov. and Wickerhamomyces gen. nov. FEMS Yeast Res 8: 939-954.

Manter, D.K., and Vivanco, J.M. (2007) Use of the ITS primers, ITS1F and ITS4, to characterize fungal abun- dance and diversity in mixed-template samples by qPCR and length heterogeneity analysis. $J$ Microbiol Methods 71: $7-14$.

Moran, N.A., McCutcheon, J.P., and Nakabachi, A. (2008) Genomics and evolution of heritable bacterial symbionts. Annu Rev Genet 42: 165-190.

Moreira, L.A., Iturbe-Ormaetxe, I., Jeffery, J.A., Lu, G., Pyke, A.T., Hedges, L.M., et al. (2009) A Wolbachia symbiont in Aedes aegypti limits infection with dengue, Chikungunya, and Plasmodium. Cell 139: 1268-1278.

Nakase, T., Jindamorakot, S., Mikata, K., Ninomiya, S., Kawasaki, H., Limtong, S., et al. (2007) Pichia koratensis sp. nov., a new ascomycetous yeast related to Pichia acaciae isolated from insect frass in Thailand. $J$ Gen Appl Microbiol 53: 345-351.

Nguyen, N.H., Suh, S.O., and Blackwell, M. (2007) Five novel Candida species in insect-associated yeast clades isolated from Neuroptera and other insects. Mycologia 99: 842858.

Noel, G.R., and Atibalentja, N. (2006) 'Candidatus Paenicardinium endonii', an endosymbiont of the plant-parasitic nematode Heterodera glycines (Nemata: Tylenchida), affiliated to the phylum Bacteroidetes. Int J Syst Evol Microbiol 56: 1697-1702.

Passoth, V., Fredlund, E., Druvefors, U.A., and Schnürer, J. (2006) Biotechnology, physiology and genetics of the yeast Pichia anomala. FEMS Yeast Res 6: 3-13.

Pérez, J., Infante, F., Vega, F.E., Holguín, F., Macías, J., Valle, J., et al. (2003) Mycobiota associated with the coffee berry borer (Hypothenemus hampel) in Mexico. Mycol Res 107: 879-887.

Rani, A., Sharma, A., Rajagopal, R., Adak, T., and Bhatnagar, R.K. (2009) Bacterial diversity analysis of larvae and adult midgut microflora using culture-dependent and cultureindependent methods in lab-reared and field-collected Anopheles stephensian Asian malarial vector. BMC Microbiol 9: 96. doi: 10.1186/1471-2180-9-96.

Rao, R.S., Bhadra, B., and Shivaji, S. (2007) Isolation and characterization of xylitol-producing yeasts from the gut of colleopteran insects. Curr Microbiol 55: 441-446.

Riehle, M.A., and Jacobs-Lorena, M. (2005) Using bacteria to express and display anti-parasite molecules in mosquitoes: current and future strategies. Insect Biochem Mol Biol 35: 699-707.

Rojas, V., Gil, J.V., Piñaga, F., and Manzanares, P. (2003) Acetate ester formation in wine by mixed cultures in laboratory fermentations. Int J Food Microbiol 86: 181-188.

Sacchi, L., Genchi, M., Clementi, E., Bigliardi, E., Avanzati, A.M., Pajoro, M., et al. (2008) Multiple symbiosis in the leafhopper Scaphoideus titanus (Hemiptera: Cicadellidae): details of transovarial transmission of Cardinium sp. and yeast-like endosymbionts. Tissue Cell 40: 231-242.

dos Santos, D.C., and Gregório, E.A. (2002) Ultrastructure of the ovariole sheath in Diatraea saccharalis (Lepidoptera: Pyralidae). Biocell 26: 229-235.

Sun, H., Ma, H., Hao, M., Pretorius, I.S., and Chen, S. (2009) Identification of yeast population dynamics of spontaneous fermentation in Beijing wine region, China. Ann Microbiol 59: 69-76.

Thompson, M.T. (1905) Alimentary canal of the mosquito. Proc Boston Soc Nat Hist 32: 145-202. 
Wang, L., Yue, L., Chi, Z., and Wang, X. (2008) Marine killer yeasts active against a yeast strain pathogenic to crab Portunus trituberculatus. Dis Aquat Organ 80: 211218.

Wang, X., Chi, Z., Yue, L., Li, J., Li, M., and Wu, L. (2007) A marine killer yeast against the pathogenic yeast strain in crab (Portunus trituberculatus) and an optimization of the toxin production. Microbiol Res 62: 77-85.
Xufre, A., Albergaria, H., Inácio, J., Spencer-Martins, I., and Gírio, F. (2006) Application of fluorescence in situ hybridisation (FISH) to the analysis of yeast population dynamics in winery and laboratory grape must fermentations. Int $J$ Food Microbiol 108: 376-384.

Zacchi, L., and Vaughan-Martini, A. (2002) Yeast associated with insects in agricultural areas of Perugia. Ann Microbiol 52: 237-244. 